\title{
Reduction of nanowire diameter beyond lithography limits by controlled catalyst dewetting
}

\author{
Yonatan Calahorra ${ }^{1,2}$, Alexander Kerlich ${ }^{1}$, Dor Amram ${ }^{3,4}$, \\ Arkady Gavrilov ${ }^{1}$, Shimon Cohen ${ }^{1}$ and Dan Ritter ${ }^{1}$ \\ ${ }^{1}$ Department of Electrical Engineering, Technion-Israel Institute of Technology, \\ Haifa 32000, Israel \\ ${ }^{2}$ Department of Materials Science and Metallurgy, University of Cambridge, \\ Cambridge CB3 0FS, United Kingdom \\ ${ }^{3}$ Department of Materials Science and Engineering, Technion-Israel Institute of \\ Technology, Haifa 32000, Israel \\ ${ }^{4}$ Department of Materials Science and Engineering, Massachusetts Institute of \\ Technology, Cambridge, Massachusetts 02139, USA \\ E-mail: yc402@cam.ac.uk, ritter@ee.technion.ac.il
}

\begin{abstract}
Catalyst assisted vapour-liquid-solid is the most common method to realize bottom-up nanowire growth; establishing a parallel process for obtaining nanoscale catalysts at pre-defined locations is paramount for further advancement towards commercial nanowire applications. Herein, the effect of a selective area mask on the dewetting of metallic nanowire catalysts, deposited within lithography-defined mask pinholes, is reported. It was found that thin disc-like catalysts, with diameters of $120-450 \mathrm{~nm}$, were transformed through dewetting into hemisphere-like catalysts, having diameters 2-3 fold smaller; the process was optimized to about $95 \%$ yield in preventing catalyst splitting, as would otherwise be expected due to their thickness-to-diameter ratio, which was as low as $1 / 60$. The catalysts subsequently facilitated $\mathrm{InP}$ and InAs nanowire growth. We suggest that the mask edges prevent surface migration mediated spreading of the dewetted metal, and therefore induce its agglomeration into a single particle. This result presents a general strategy to diminish lithography-set dimensions for NW growth, and may answer a fundamental challenge faced by bottom-up nanowire technology.
\end{abstract}

Submitted to: J. Phys. D: Appl. Phys. 


\section{Introduction}

Semiconducting nanowires (NWs) have gained significant attention for the past two decades as building blocks for future electronics, photonics, sensing applications, and as a platform for fundamental physics research [1-8]. Generally, bottom-up NW growth is realized either using a metal catalyst, which can be of a foreign material, or an element of the NW compound, or by catalyst-free methods, which are usually based on a selectivearea scheme [9-12].

The most common growth method is by a foreign metal catalyst, since it is relatively easy to implement (as will be discussed below in detail), and it also provides a mean to grow small diameter NWs. A potential shortcoming of this growth scheme is that catalyst material may penetrate the NW, and affect its electronic and optical properties; e.g., gold is the most common catalyst material, and it is known to create deep-level traps in Silicon [13]. A possible solution for this problem is using catalysts of materials which are compatible to the semiconductor processing technology. Indeed, palladium, nickel, copper and more metals were used to catalyze both III-V and silicon NWs. An extensive review regarding non-gold NW catalysts has recently been published by Dick and Caroff [11].

The true challenge faced by catalyst assisted methods, is the lack of a large-scale, spatially pre-defined, systematic growth process of sub-100 nm NWs, which remains a barrier for commercialization of NW applications; photolithography, semiconductor industry's most common processing tool, cannot easily produce features of such size. Herein we demonstrate the ability to significantly (2-3 fold) reduce the critical dimensions for NW growth, which are usually dictated by the catalyst lithography process. Potentially, this will allow photolithography based growth of sub-100 nm metalcatalyzed NWs; such capabilities are fundamental to the effort of advancing electronic NW device applications.

Nanoimprint techniques show great potential for rapid nano-scale lithography. Although not as established as e-beam lithography (EBL), nanoimprint has been successfully used to facilitate NW growth; both catalyst assisted and catalyst-free/selfcatalyzed NWs have been demonstrated [14-17]. In any case, the paradigm presented in this work is applicable to nanoimprint, as well as e-beam and photo lithography, therefore we shall not consider it in particular further-on.

\subsection{Nanowire catalyst preparation methods}

There are three approaches to create metallic NW catalysts, sorted here by increasing process complexity: i) splitting (by dewetting) of a previously evaporated continuous metallic layer; ii) dispersing metallic nanoparticles on the growth substrate; iii) lithography and lift-off (usually e-beam lithography - EBL) [9, 12,18-20]. The thermal induced dewetting of a pre-deposited metallic layer, is the simplest method; however, poor control over NW location and catalyst size is usually obtained. Using nanoparticles 
as catalysts increases process complexity but allows greater control of catalyst size, nonetheless, the resulting NW positions are still random. For further details, see the aforementioned references.

The lithography based process includes two steps: first, the location and lateral size are determined, and second, a metallization step follows, where the evaporated catalyst thickness is defined. In cost and efficiency terms, EBL catalyst definition is rated considerably low compared to definition by metallic layer dewetting or by nanoparticles. However, it offers precise control over catalyst location, and lower particle diameter variance; therefore it is favourable for systematic NW growth study, and is probably the only option for practical applications which require location control. Should a rapid sub$100 \mathrm{~nm}$ lithography process become available, significant advances in NW technology are possible.

Herein, we report on the effect of a selective area mask on the dewetting process of large-diameter, disc-like, metallic catalysts, in growth methods combining the two approaches. By confining the catalyst inside a mask opening, considerable reduction of NW diameters was obtained, compared to the initial catalyst diameter - set by the lithography process. The induced agglomeration was not possible without the presence of the selective area mask.

\section{Experimental Methods}

InP and InAs NWs were grown by metal-organic molecular beam epitaxy (MOMBE) utilizing native-oxide selective area (NOSA) growth [21], and selective area vapourliquid-solid (SAVLS) [22, 23]; SAVLS has been used to study entangled and single photon emission from NW embedded quantum dots [24, 25]. Although details of the growth appear in the references, specifics relevant to the results will be discussed in particular below (Fig. 1a). Briefly, InP or InAs (111)B oriented n-type wafers were used as growth substrates; for SAVLS, a $15 \pm 1 \mathrm{~nm} \mathrm{SiN}_{\mathrm{X}}$ layer, deposited by plasma enhanced chemical vapour deposition, covered the substrates. E-beam lithography was performed to realize large-diameter (120-450 nm), as well as standard-sized (about 40 $\mathrm{nm}$ ), catalysts, by a standard lift-off procedure; this included PMMA coating, EBL, wet etch prior to evaporation (BOE for SAVLS, $\mathrm{H}_{2} \mathrm{SO}_{4}: \mathrm{H}_{2} \mathrm{O}$ 1:10 for NOSA) and e-beam based evaporation of gold. Gold deposition was performed in a high-vacuum e-beam evaporator (Airco Temescal FC 1800), at about $0.3 \mathrm{~nm} / \mathrm{sec}$ with $\pm 1 \mathrm{~nm}$ accuracy. The duration of BOE treatment determines the nitride mask opening, while the gold catalyst diameter is determined by the EBL process (Fig. 1b, Fig. 1c).

For every EBL process, several thicknesses of evaporated gold were used - thus taking advantage of the two-dimensional parameter space available for lithography based catalyst definition. Nanowire growth temperature was $420-450{ }^{\circ} \mathrm{C}$, and an insitu, 1-5 minute, pre-growth heating treatment, with temperatures $30-80{ }^{\circ} \mathrm{C}$ higher, was employed. Nanowire length and diameter characterization was performed by scanning 


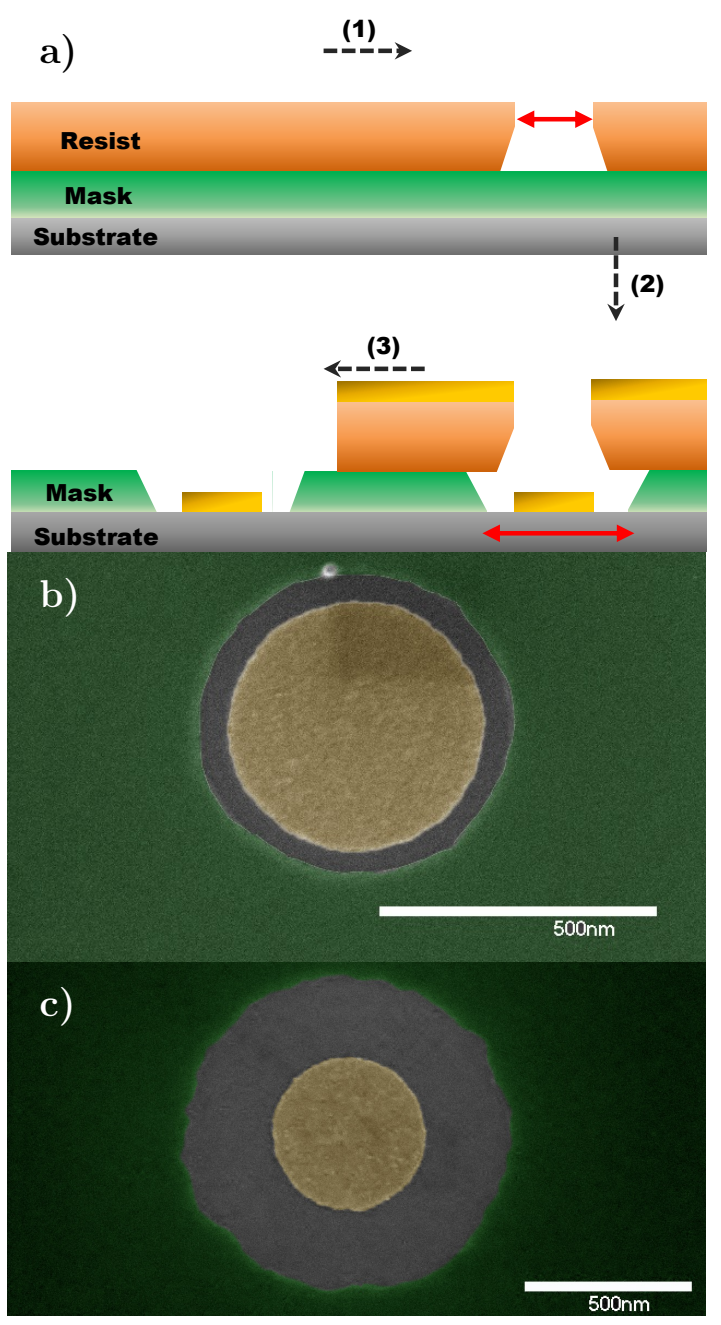

Figure 1: a) Fabrication process schematic: a substrate covered with a selective area mask; (1) EBL and develope, this stage determines the initial catalyst diameter (solid red arrow); (2) mask etch and metalliation, this stage determines the mask opening diameter (solid red arrow - notice the difference between [b] and [c]) and the initial catalyst thickness; (3) metal lift-off, ready for MOMBE. b,c) The process results: topview, false-colored, scanning electron micrographs of post metal-deposition catalysts on InAs ( $8 \mathrm{~nm}$ metallization, diameter $450 \mathrm{~nm}$ ). The $\mathrm{SiN}_{\mathrm{X}}$ mask has been over-etched in $[\mathrm{c}]$.

electron microscopy (SEM, Hitachi S4700); an example for a successful NW growth with standard catalyst diameters (in terms of EBL defined dimensions) is shown in Fig. 2 inset. 


\section{Results and discussion}

Figure 1a shows a schematic of the catalyst fabrication process (discussed in detail in Sec. 2), and corresponding results. As mentioned above, in lithography based processes, the catalyst thickness and diameter are decoupled, and controlled separately; allowing for very low thickness to diameter ratios to be examined. We explore the effects of combining catalyst assisted methods with a selective area mask on NW growth by catalysts of low thickness to diameter ratio. Figures $1 \mathrm{~b}, \mathrm{c}$ show the resulting disc-like catalyst (on InAs in this case) [26]. Notice the different annular openings - a result of different mask etching time using the same lithography.

\subsection{Agglomeration of small diameter catalysts}

In the native-oxide selective area (NOSA) NW growth regime, the native oxide is not removed off the substrate prior to growth, leaving a $\sim 1 \mathrm{~nm}$ layer of indium and phosphorous oxides; this layer prevents nucleation on the surface and induces long-range surface diffusion of the metal-organic precursors towards the catalyst [21]. Recently we have found that using different sets of lithography diameter and metallization thickness, to realize NWs of similar diameters in this growth regime, may result in significantly different NW lengths [27]; here however, we are mainly concerned with the resulting NW diameter. For further information about NW lengths and the effect of different pinhole diameters on NW growth see reference 27.

Figure 2 shows NW diameter vs. metallization thickness for a $\sim 40 \mathrm{~nm}$ diameter (measured by SEM prior to growth) pinhole, where the metallization was varied from 1 to $25 \mathrm{~nm}$; each data point is the average of at least $10 \mathrm{NWs}$, as measured by SEM (see Fig. 2 inset and Ref. [21] for typical growth results). Generally, with increased metallization thickness, an increase in NW diameter was found. Interestingly, the thinnest metallizations resulted in NWs which were of diameters smaller than the initial catalyst diameter; moreover, the catalysts discussed here did not suffer any effect of splitting, even at the most-thinnest metallizations. The yield of vertical NW growth in these experiments was close to $100 \%$.

Mårtensson et al. have found that for lithography based catalysts, a minimum $1 / 3-1 / 6$ thickness to diameter ratio is required to prevent catalyst splitting [28]; in other cases ratios of $1 / 15$ yielded adequate results (6 nm thickness, $100 \mathrm{~nm}$ diameter, by nanoimprint), following surface treatment optimization [15]. In the experiments reported here however, using the simple NOSA scheme allowed avoiding the splitting effect and opened-up the possibility of tuning the resulting NW diameter with extreme thickness to diameter ratios; we note that the thinnest catalysts used (namely 1 and $2 \mathrm{~nm}$ ) correspond to $1 / 40$ and $1 / 20$ thickness to diameter ratio, which is considerably smaller than the ratios reported as mandatory for successful NW growth in the literature.

We apply a hemispherical model (dotted line in Fig. 2) to describe the catalyst 


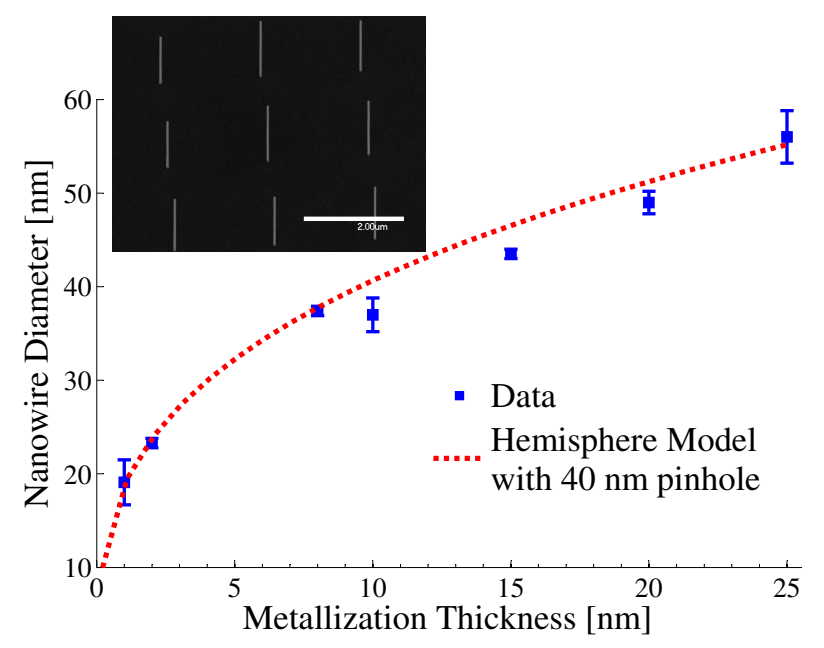

Figure 2: Nanowire diameter vs. catalyst metallization thickness, for NWs grown by NOSA (blue dots); the error bars correspond to the standard deviation in the measurement. The dashed red line shows the hemispherical model calculation following Eq. 1-2. The inset shows tilted SEM captures of typical growth results.

shape during the growth, in order to quantify the relation between the NW diameter and the pinhole diameter and metallization used. If so, the diameter, $D_{N W}$, is related to the catalyst volume, $V_{\text {cat }}$, by

$$
D_{N W}=2\left(\frac{6 V_{\text {cat }}}{4 \pi}\right)^{1 / 3}
$$

and the catalyst volume is given by

$$
V_{\text {cat }}=\frac{\pi r_{\text {pinhole }}^{2} T_{M}}{1-\beta}
$$

where $r_{\text {pinhole }}$ is the pinhole radius, $T_{M}$ is the metallization thickness, and $\beta$ is the ratio of non-gold elements in the catalyst during growth. For InP and InAs NWs there is a non-negligible amount of indium in the gold catalyst; we follow the report by Fröberg et al. and use $\beta_{\text {InP }}=0.45$ and $\beta_{\text {InAs }}=0.35$ [29]. Using equations 1 and 2 with $r_{\text {pinhole }}=20$ $\mathrm{nm}$ and $\beta_{\text {InP }}$, the experimental results were reproduced quite well, indicating that the basic understanding of the process which the catalyst undergoes during growth is valid.

These results are not exclusive to growth by the NOSA regime, and we have observed very similar results in growth by selective area vapour-liquid-solid (SAVLS); we do not pay specific attention to that since the focus of this research is the controlled agglomeration of large-diameter catalyst, as discussed below.

\subsection{Agglomeration of large diameter catalysts}

A natural subsequent step was to repeat the NOSA experiment with much larger lithography pinholes, which could potentially be prepared by photolithography; 


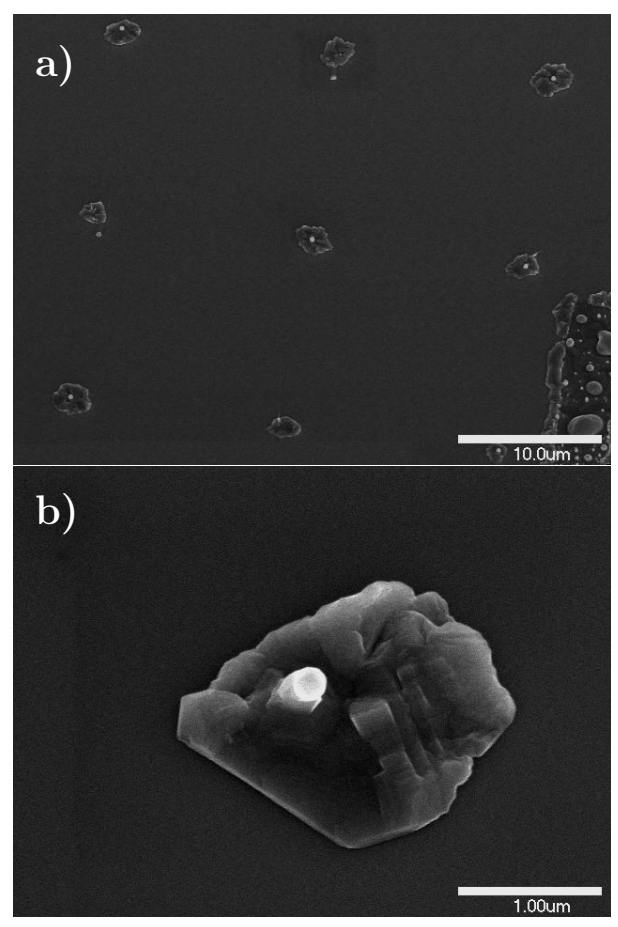

Figure 3: a) Scanning electron micrographs of large-diameter ( $\sim 450 \mathrm{~nm})$ growth results obtained by NOSA; b) zoom-in to one of the growth sites in [a].

therefore, catalysts with pinholes ranging from 120-450, and a metallization of 10 $\mathrm{nm}$, were prepared by EBL. However, this experiment did not yield satisfying growth results: although only a small fragment of the catalysts experienced splitting, most catalyst resulted in very short NWs with large, bulky, bases, of diameter exceeding that of the original pinhole; figure 3 shows an example of this growth. If so, although allowing significant catalyst size reduction for initially small pinhole diameters, NOSA growth conditions did not facilitate successful size reduction for catalysts originating in photolithography-scale pinholes. The origin of this result could be the long diffusion length of group-III precursors on the native oxide, resulting in a high planar growth rate on surface exposed by catalyst agglomeration.

In SAVLS method, a growth-stop layer ( $\mathrm{SiN}_{\mathrm{X}}$ in this report) is deposited on the growth substrate, prior to lithography and metal deposition; next, the lithography pattern is used both as an etch mask, and as a metallization mask, thus the catalysts are deposited inside pinholes etched in the mask (Fig. 1). Nanowire growth is facilitated only by the catalysts inside the pinholes, while metal-organic growth precursors scatter off the mask, and the scattered material fraction impinging the NW sidewall, contributes to growth $[22,23]$. The listed references and subsequent publications include thorough analysis of this growth method, however its application to large diameter (photolithography scale) catalysts has not been reported.

Pinholes of about 120-450 nm were realized by EBL on InP and InAs substrates. Metallizations of 4 and $8 \mathrm{~nm}$ for InP NWs, as well as 8 and $20 \mathrm{~nm}$ for InAs NWs were 
Table 1: Large-diameter catalysts of $8 \mathrm{~nm}$ on InP;

$D_{1}=130 \mathrm{~nm}, D_{2}=230 \mathrm{~nm}, D_{3}=340 \mathrm{~nm}, D_{4}=450 \mathrm{~nm}$

\begin{tabular}{|c|c|c|c|c|c|c|c|c|}
\hline \multirow[t]{2}{*}{ Growth \# } & \multirow{2}{*}{$\begin{array}{c}\text { Temp. }\left[{ }^{\circ} \mathrm{C}\right] \\
\text { Growth } \\
\text { (Pre-growth) }\end{array}$} & \multicolumn{4}{|c|}{$\begin{array}{l}\text { Non-split yield } \\
\left(\text { Total yield }^{b}\right)[\%]\end{array}$} & \multirow{2}{*}{$\begin{array}{c}\text { Annular } \\
\text { opening } \\
{[\mathrm{nm}]}\end{array}$} & \multirow{2}{*}{$\begin{array}{l}\mathrm{H}_{2} \mathrm{SO}_{4} \\
\text { etching }\end{array}$} & \multirow[t]{2}{*}{ See Fig } \\
\hline & & $D_{1}$ & $D_{2}$ & $D_{3}$ & $D_{4}$ & & & \\
\hline 1. Non-SA ${ }^{c}$ & $420(530)$ & $33(33)$ & $13(6)$ & $6(0)$ & $0(0)$ & - & No & $4 \mathrm{~d}-\mathrm{f}$ \\
\hline 2. SAVLS & $420(530)$ & $100(73)$ & $86(73)$ & $33(13)$ & $33(13)$ & $45-50$ & No & $4 a-c$ \\
\hline 3. SAVLS & $420(455)$ & $67(67)$ & $72(72)$ & $67(33)$ & $0(0)$ & $45-50$ & Yes & - \\
\hline 4. $S A V L S+I$ & $420(455)$ & $92(46)$ & $80(80)$ & $78(78)$ & $78(78)$ & $\sim 0^{d}$ & No & $5 \mathrm{c}$ \\
\hline 5. SAVLS $+\mathrm{NOSA}^{d}$ & $450(455)$ & $96(47)$ & $94(83)$ & $94(89)$ & $93(93)$ & $\sim 0^{d}$ & No & $5 \mathrm{a}, \mathrm{b}$ \\
\hline
\end{tabular}

$a$ - Yield of single particles per-catalyst

$b$ - Yield of single vertical NWs per-catalyst

$c$ - The $530{ }^{\circ} \mathrm{C}$ treatment removes the native oxide, rendering the growth non-selective in the absence of a $\mathrm{SiN}_{\mathrm{X}}$ mask

$d$ - The $455{ }^{\circ} \mathrm{C}$ treatment does not remove the native oxide, leaving the annular opening covered by a selective mask

used to realize the disc-like catalysts; notice the thickness-to-diameter ratios examined in this experiment, which are as small as $1 / 100$. We shall first focus on a set of growth experiments performed on $8 \mathrm{~nm}$ metallization on InP substrates, to explore the effect of growth parameters on the yield of single vertical NWs from thin, disc-like, large diameter gold catalysts. The remainder of the results, with specific consideration of the catalyst-diameter reduction, will be subsequently presented.

Table 1 lists the growth conditions and the results for $8 \mathrm{~nm}$ metallization on $\mathrm{InP}$ substrates. Two yield parameters are considered: first, the non-split yield, describing the fraction of catalysts which underwent successful agglomeration into a single particle; and second, the total yield, describing the fraction of catalysts resulting in single vertical nanowires. High, medium and low non-split yield values are highlighted in green, yellow and red, correspondingly. The diameters examined were about 130, 230, 340 and 450 $\mathrm{nm}$. The statistics were taken from 18-20 catalyst locations per data point in the table.

All growth substrates were identical, except substrate 1, which did not include the SiN layer (identical to growth substrates prepared for the NOSA experiment reported above). The parameters examined were growth and pre-growth annealing temperatures and the influence of an $\mathrm{H}_{2} \mathrm{SO}_{4}: \mathrm{H}_{2} \mathrm{O}$ dip prior to growth; the precursor flow rates and growth time (50 minutes) were not changed. Dipping the sample in $\mathrm{H}_{2} \mathrm{SO}_{4}: \mathrm{H}_{2} \mathrm{O}$ immediately before insertion to the growth chamber, removes the native oxide from the annular opening (which is related to the duration of the BOE dip performed prior to metallization). Another option to remove the native oxide from the annular opening is by a pre-growth heating treatment higher than $480{ }^{\circ} \mathrm{C}$ [21].

Comparing growth experiments 1 and 2, directly probes the effect of the $\mathrm{SiN}_{\mathrm{X}}$ mask with an exposed annular opening, on the agglomeration of large-diameter disc- 


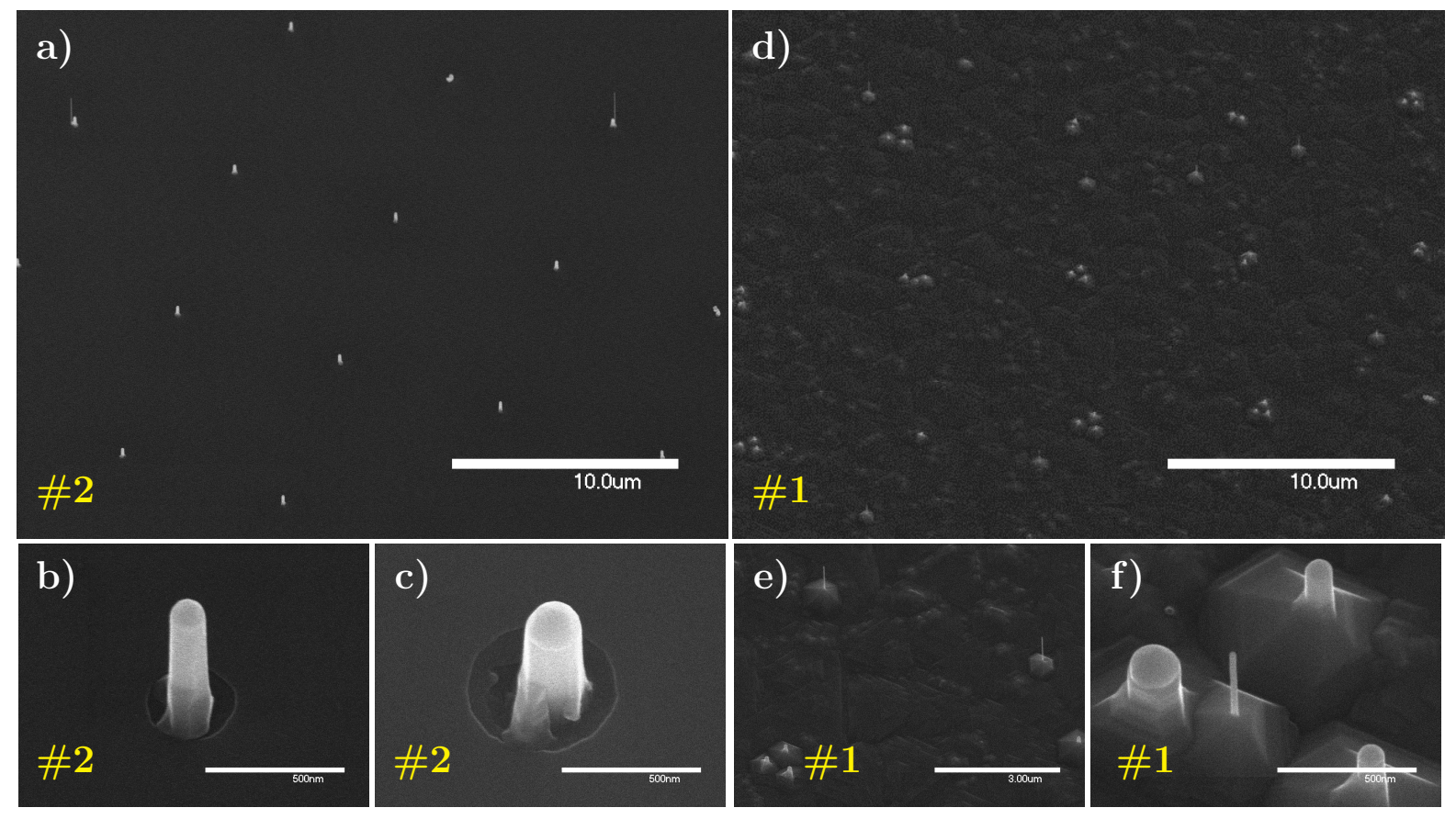

Figure 4: $30^{\circ}$ tilted scanning electron micrographs of: a-c) Growth \#2 - SAVLS; d-f) Growth \#1 - non selective. [a,b] and [d,e] show results for $D_{2}=230 \mathrm{~nm}$ catalysts on the two samples; [c] and [f] show growth from catalysts of $D_{4}=450 \mathrm{~nm}$. Lower scale bars are $500 \mathrm{~nm}$ in $[\mathrm{b}, \mathrm{c}, \mathrm{f}]$ and $3 \mu \mathrm{m}$ in $[\mathrm{e}]$.

like catalysts. The trend is clear - the existence of a selective area mask, even if not directly around the catalyst (due to the annular opening) resulted in significant increase of both yield parameters. Figures 4a,b,d,e demonstrate this for the $230 \mathrm{~nm}$ catalysts exhibiting a 6 -fold increase in non-split yield; while figures $4 \mathrm{c}, \mathrm{f}$ show the growth results of single $450 \mathrm{~nm}$ catalyst in the selective and non-selective schemes, showing a single nanowire growing from the mask opening, while at the unmasked catalyst location, splitting occurred, resulting in spreading of the catalyst fragments - most noticeably, in an area much larger than the original catalyst diameter.

Next, comparing growth experiments 3-5, shows the effect of the native oxide in the annular opening (experiments 3,4), and that of the growth temperature (experiments $4,5)$. If so, by not removing the native oxide, hence combining the SAVLS method with a NOSA annular opening, the yield increases dramatically. Specifically, in the largest diameter examined, from zero yield to about $80 \%$ in both parameters, with all the successfully agglomerated catalysts resulting in vertical NWs. An example of these results can be seen in Fig. 5a-c. Standing out is the reduction in vertical yield in the smallest diameters, despite the increase in non-split yield (The difference between nonsplit and growth yield values of $D_{1}$ in table 1 rows \#4,5 - no figure is shown); we believe this result is related to the increased intake of indium due to the efficient surface diffusion of the group-III precursor on the NOSA surface [21], which may impede vertical growth $[9,30]$. Considering the high yield of vertical NWs in the larger catalysts, it is 
not likely that there is a fundamental barrier for growth in these conditions.

Growth experiments 4 and 5 show the effect of the growth temperature. Growth

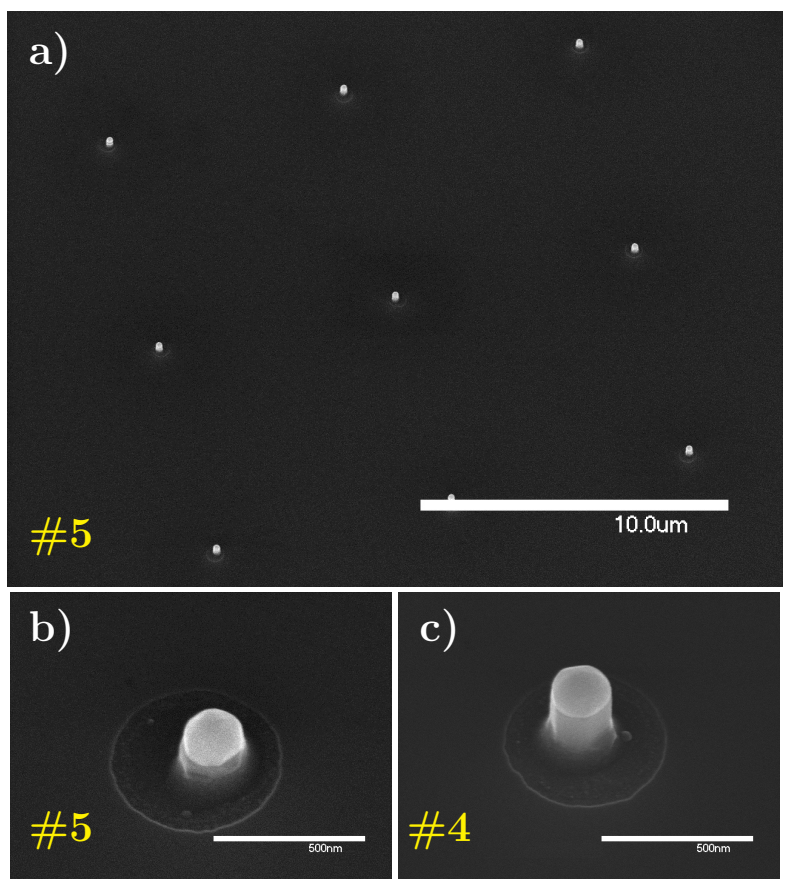

Figure 5: $30^{\circ}$ tilted scanning electron micrographs of: a,b) Growth \#5; c) Growth \#4 for $D_{4}=450 \mathrm{~nm}$. The difference in NW height results from the different growth temperature. Lower scale bars are $500 \mathrm{~nm}$.

at $450{ }^{\circ} \mathrm{C}$ resulted in improved yield compared to growth at $420{ }^{\circ} \mathrm{C}$, when all other parameters are similar; non-split yield was close to $95 \%$ in all diameters, and the vertical yield was in accordance (except for the abnormality in the smallest diameters, as discussed above). We suggest that this is an outcome of the growth process involving annealing: when the catalyst is heated up to $455^{\circ} \mathrm{C}$ the indium concentration and chemical potential reaches equilibrium with the surroundings; next, when the temperature is dropped toward growth temperature, different concentrations and chemical potentials are reached - probably influencing the contact angle and catalyst diameter. Indeed, we have generally observed slightly thinner and longer NWs for growth at $420^{\circ} \mathrm{C}$. It is also possible that during this process the catalyst is more likely to split due to the changes in its composition. Contrary, when the cool-down is merely $5{ }^{\circ} \mathrm{C}$ (as in growth experiment 5) less splitting occurs; this point, however, requires further inquiry.

If so, the reported set of experiments points out to several conclusions: first and foremost, the presence of a selective area mask around the catalyst, induces its agglomeration into a single particle, which then facilitates NW growth with diameters significantly smaller than the original pinhole dictated by lithography (e.g., from 450 to $200 \mathrm{~nm}$ in $8 \mathrm{~nm}$ metallization); second, nearly perfect yield of single vertical nanowires per large-diameter (with less than 1/50 thickness to diameter ratio) catalyst site, is 
achievable by correctly tuning the process parameters; third, the process yield increases with better confinement of the catalyst by the mask (a native oxide covering the annular opening in this case [31]).

Next we turn to a quantitative analysis of the agglomeration, comparing the final NW diameter to the original pinhole diameter, for InAs and InP NWs of different metallization thicknesses. To do so we have only considered catalysts which agglomerated with a relatively high yield. Figure 6 summarizes the growth results obtained by SAVLS with large catalysts [32]; the predicted diameter values according to Eq. 1 are plotted as well, no fitting was performed. Generally, the thin discs followed the hemispherical trend on both materials, and resulted in catalysts with diameters 2-3 times smaller. A shape slightly different than hemisphere, or a different intake of indium into the catalyst, could be the reason for the discrepancies between model and experimental data for the $20 \mathrm{~nm}$ gold discs deposited on InAs. The $120 \mathrm{~nm}$ data point of the $20 \mathrm{~nm}$ metallization in Fig. 6a is missing due to unsuccessful processing; while the two wider data points of the $4 \mathrm{~nm}$ metallization in Fig. $6 \mathrm{~b}$ are missing since these catalysts did not result in single NWs and experienced splitting. We conclude that in these conditions (similar to growth experiment 4), a thickness-to-pinhole ratio of 1/60 is the limit for systematic use of this method to reduce lithography based InP NW catalysts diameters. The figure inset shows an example of an InAs NW grown in this process.

The results presented in Fig. 6, demonstrate the generality of the process, indicating it could be applied to other material systems, and perhaps be used to defy the limits of photo-lithography for the bottom-up realization of nanomaterials. It should be noted that this process suffers from a limitation on the NW area fill-factor available, since inherently, a large part of the area initially covered by metal does not facilitate growth; e.g. for a 3-fold reduction of catalyst size the fill factor may only be as high as $\sim 10 \%$. This should be taken into consideration when thinking about possible applications.

\subsection{Dewetting: splitting vs. agglomeration}

The main finding presented in this paper is that metallic thin-film dewetting can be engineered to result in a single-particle agglomeration, although the deposited layer dimensions suggest it should undergo splitting; this is done be metal deposition inside predefined physical barriers (pits). Therefore we conclude that the barriers induce a process which counter-acts the expected splitting, and enables realization of catalysts with diameters considerably smaller than the original particle diameter (see Fig. 4c,f).

The underlying mechanism for thin-film dewetting is energy minimization, mediated by surface diffusion of atoms to reach the equilibrium shape [33-36]. The quantitative analysis of this phenomenon is highly complex and has to take into account the materials in question, system temperature, thermal expansion coefficients, time evolution, and also issues such as the crystalline nature of the substrate, and the interaction between the ad-layer and the substrate [37-40]. We will provide a semi-quantitative discussion of 

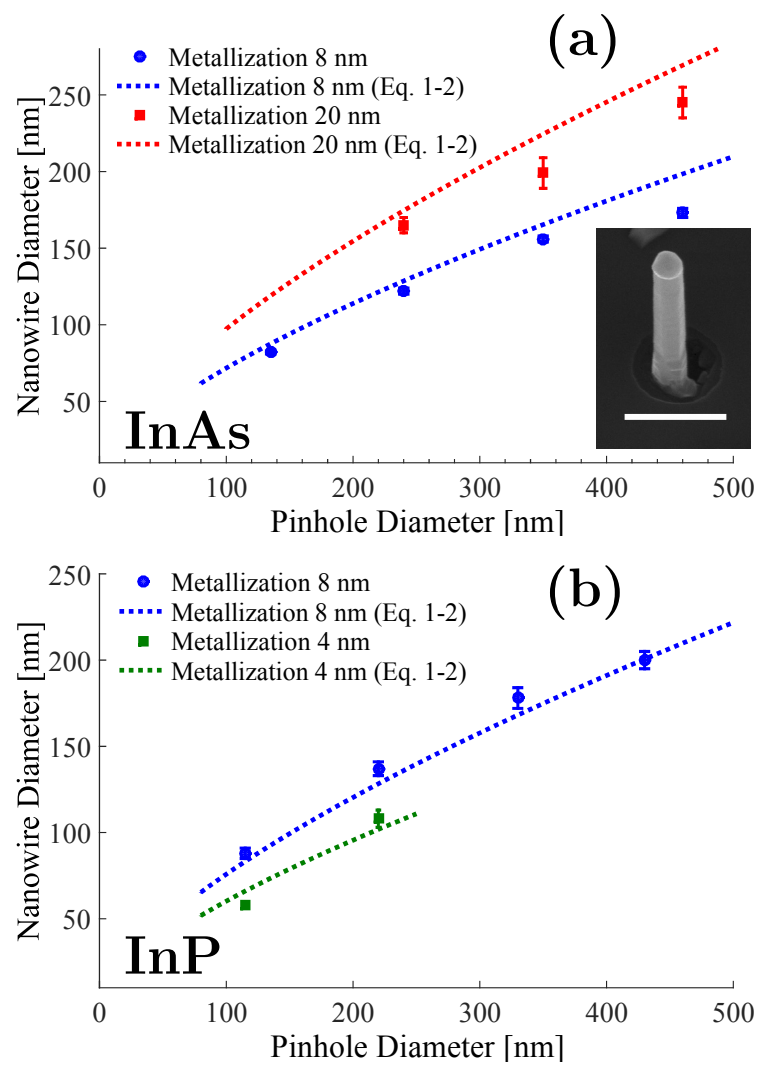

Figure 6: Nanowire diameter vs. resist pinhole diameter for (a) InAs NWs; (b) InP NWs. Different data sets correspond to different metallization thicknesses used (20 and $8 \mathrm{~nm}$ in [a], 8 and $4 \mathrm{~nm}$ in [b]), and the dashed lines are predictions based on the hemispherical model (Eq. 1 and 2). The inset shows a $140 \mathrm{~nm}$ InAs NW grown from a $350 \mathrm{~nm}$ catalyst disc at $20 \mathrm{~nm}$ metallization; scale bar is $500 \mathrm{~nm}$.

the results presented here, in light of major effects in thin-film dewetting.

Generally, two main guidelines for energy minimization dictate the dewetting process and results, considering the geometry and the materials in question: 1) the total surface/interface energy of the system increases with increasing curvature, at a fixed volume; 2) the equilibrium contact angle balances the interfacial tensions between the phases (Young-Laplace equation). The former indicates that sharp features such as corners, peaks and valleys would be smoothed during dewetting, and that wetting of steps and corners is not energetically favored; the latter defines the shape of the layer termination. These guidelines do not, however, determine whether the layer will agglomerate into a single particle, or split into several particles. It is important to note that in the context of NW growth, there is usually significant interaction between the catalyst and the substrate, manifested by a significant intake of substrate material into the catalyst [29,41,42]; therefore in addition to the effects mentioned above, the reaction between the catalyst and the substrate may effect the conditions for the dewetting evolution. 
Typically, the dewetting process involves an initial nucleation stage, where defects and grain boundaries mediate the creation of pores (and subsequently islands) in the film, according to the guidelines mentioned above; next, depending on time and temperature, surface diffusion of atoms, and surface reactions, will change the size distribution, location and morphology of the islands. The thermodynamically favoured result of the process is a single particle, containing all the film material - due to surface energy minimization; practically, however, the result will usually be some distribution of particles on the surface. The initial distribution of defects and subsequent pores will determine the final result.

Recalling growth experiments 1-2 and 3-4, the results point to the role of the mask in inducing agglomeration; moreover, as the mask becomes closer to the catalyst (by the native oxide covering the annular opening in the second set of experiments), singleparticle agglomeration becomes more efficient. Considering that the catalyst deposition was identical in all cases, we conclude that the mask affects the second stage in the evolution of the catalyst - the movement of the islands created from the original catalyst. But what is the nature of this effect?

Taking a closer look at Fig. 4d-f, it is clear that when splitting occurs, the catalyst fragments end up spread across an area which is much larger than that of the deposited catalyst; similarly, even in those cases where the mask did not manage to prevent splitting (Fig. 5e,f), catalyst fragments were found close to the opening periphery, indicating some level of spreading. Therefore, we suggest that preventing the catalyst fragments from spreading, is the key to inducing their agglomeration.

Due to material and energy considerations, the catalyst fragments are highly unlikely to wet the mask layers; specifically, with surface energies of 1.2 and $0.046 \mathrm{~J} \cdot \mathrm{m}^{-2}$, gold and $\mathrm{SiN}_{\mathrm{X}}$ exhibit poor wetting [43]. Evidently, even the native oxide layer, which is less than one nanometer thick, acts to prevent wetting, and subsequently splitting (Fig. 3). Next, considering that the catalyst fragments tend to spread across the surface in a motion mediated by adatom diffusion and catalyst-substrate reactions, it stands to reason that preventing the motion of the fragments outside a given area, would significantly increase their chances to come across each other and make adatom diffusion and exchange of material between fragments easier. Although the surface diffusion coefficient itself is not the most relevant parameter to describe the rate of dewetting (rather, it is the product of that coefficient and the surface density of mobile atoms), it indeed does provide partial indication on mass transfer rate. At the temperatures employed in this work, the Au surface diffusion coefficient, $D_{s}$, is of the order of $10^{-16}$ $\mathrm{m}^{2} \mathrm{~s}^{-1}$ [44]. For a heat treatment of only $1 \mathrm{~min}$ at $400 \mathrm{C}$, the characteristic diffusion length, $X=\sqrt{D_{s} t}$, is already roughly $200 \mathrm{~nm}$; this length is of the order of the mask pinhole size. It should be mentioned that the diffusion length is a very crude underestimate, since it is derived simply from random walk theory, while the driving force for dewetting is capillary. This capillary driving force is very high during the initial period of dewetting, when the curvature is highest. Therefore, it is likely that most of the catalyst film agglomerated into particles during the first few moments of 
the heat treatment, after which these particles change their shape slowly en route to their equilibrium crystal shape. In nanocrystalline thin films this process is facilitated by the presence of a large fraction of grain boundaries, which are preferred sites for hole formation [45]; indeed, even Au films of $25 \mathrm{~nm}$ in thickness and heat treated at $400 \mathrm{C}$ form micrometer-sized holes after similar durations [46]. Since the dewetting rate is proportional to the curvature (and therefore inversely proportional to the film thickness [36]), the $8 \mathrm{~nm} \mathrm{Au} \mathrm{films} \mathrm{employed} \mathrm{in} \mathrm{this} \mathrm{work} \mathrm{are} \mathrm{expected} \mathrm{to} \mathrm{agglomerate}$ much faster. Furthermore, the fixed boundaries for a catalyst fragment at the mask edges may also induce a driving force for agglomeration as a mean to reduce its surface energy. If so, the proposed route for efficient catalyst agglomeration starts with the reduced chance of catalyst fragments to wet the mask edges, resulting in an increased probability of adatom diffusion, and of the fragments to reduce their surface energy within the pinhole - leading to an overall tendency of the catalyst to agglomerate as the mask opening confinement increases.

\subsection{From metallic thin-films to nanoparticles - an outlook}

Finally, we discuss recent advances in the ability to control the dewetting processes, and significantly reduce the dimensions of thin metallic films; a comparison is made in the context of VLS NW growth. The Control of dewetting procedure outputs is challenging, and various concepts have been explored. For example, the the effect of substrate crystalline structure and orientation was examined by Ye and Thompson [38]; they have shown that the original template orientation, relative to the substrate, could significantly impact shape of the dewetted structure. Dewetting of layers on templated substrates was examined as well; $\mathrm{Oh}$ and co-workers, have studied the dewetting of cobalt films deposited on selectively etched oxidized silicon substrates, to find that by pre-patterned etching of the substrate, the final shape of the dewetted film could be controlled to prefer specific locations inside etched inverted pyramids; thus manifesting control over dewetting down to a single particle level. Interestingly, in the right conditions, dewetting can even result in the formation of metallic NWs directly from the thin-film [47].

Sundar et al. presented a method to reduce the mean diameter and the variation of gold particles created by dewetting. A platinum foil deposited on top of the gold film acted to consume some of the dewetted gold, in a selective process which singles out the larger particles [48]. Farzinpour et al. have also used a two-material approach to control dewetting; by depositing the layer of interest on top of an antimony layer. During annealing the antimony layer sublimates, from the periphery towards the center, leaving an agglomerated particle of the second deposited layer [49]. Pan et al. used a thin-film of silver to grow InAs NWs on Silicon; they have demonstrated that adding a low-temperature anneal step, preceding the common high-temperature one (usually employed to dewet the metallic film and remove substrate surface oxide), resulted in high-density, small-diameter NWs. They suggest that the low-temperature anneal 
Table 2: Nano-particle by controlled thin-film dewetting

\begin{tabular}{|c|c|c|c|c|c|}
\hline Method & $\begin{array}{l}\text { Relative process } \\
\text { complexity }\end{array}$ & $\begin{array}{c}\text { Max. } \\
\text { Temperature }\end{array}$ & Achievements & $\begin{array}{l}\text { Implications on } \\
\text { NW growth }\end{array}$ & $\begin{array}{c}\text { Nanowires } \\
\text { demonstrated }\end{array}$ \\
\hline $\begin{array}{l}\text { Top Pt } \\
\text { cover [48] }\end{array}$ & $\begin{array}{l}\text { Additional layer } \\
\text { deposition and } \\
\text { removal }\end{array}$ & $600-1000{ }^{\circ} \mathrm{C}^{b}$ & $\begin{array}{l}\text { NP size and } \\
\text { variation } \\
\text { reduction }\end{array}$ & $\begin{array}{l}\text { Possible different } \\
\text { catalyst substrate } \\
\text { interaction }\end{array}$ & No \\
\hline $\begin{array}{c}\text { Dynamic } \\
\text { templating } \\
{[49]}\end{array}$ & $\begin{array}{c}\text { Additional layer } \\
\text { deposition; } \\
\text { heating }\end{array}$ & $600-1000{ }^{\circ} \mathrm{C}$ & $\begin{array}{l}\text { Very high } \\
\text { aspect ratio } \\
\text { coalescence }\end{array}$ & $\begin{array}{l}\text { Antimony residue; } \\
\text { substrate heating }\end{array}$ & No \\
\hline $\begin{array}{c}\text { Low } \\
\text { temp. an- } \\
\text { nealing }[50]\end{array}$ & Negligible & $\begin{array}{l}\text { Same as } \\
\text { control }\end{array}$ & $\begin{array}{l}\text { NP size and } \\
\text { variation } \\
\text { reduction }\end{array}$ & $\begin{array}{l}\text { Possible different } \\
\text { catalyst substrate } \\
\text { interaction }\end{array}$ & Yes \\
\hline This work & $\begin{array}{c}\text { Additional layer } \\
\text { deposition and } \\
\text { etch }^{c}\end{array}$ & $\begin{array}{l}\text { Same as } \\
\text { control }\end{array}$ & $\begin{array}{l}\text { NW diameter } \\
\text { 2-3 times } \\
\text { smaller than } \\
\text { lithography }\end{array}$ & $\begin{array}{l}\text { Limited aspect } \\
\text { ratio }\end{array}$ & Yes \\
\hline
\end{tabular}

$a$ - Removal after NPs are formed

$b$ - Not necessarily higher than the control process

$c$ - Etching prior to catalyst deposition

step acts to suppress Ostwald ripening of the droplets [50]. Table 2 compares the approach presented here and the above methods, with specific considerations regarding NW growth.

The first two methods, since they involve the deposition of an additional metallic layer, may result in unwanted residues in the NW catalyst, or unwanted catalystsubstrate chemical interactions, due to different temperature ranges used; indeed, antimony was found to impede SiNW growth [51]. Nonetheless the successful control over dewetting properties is promising in terms of NW growth. The later, lowtemp. annealing, and the work presented here, do not require significant complications compared to the standard process, and indeed NW growth has been demonstrated.

In is noteworthy that contrary to the other methods surveyed, the lowtemp. annealing method is essentially about counteracting agglomeration and achieving a uniform metallic layer splitting by dewetting. This is a significant distinction, since combining two of the above methods might result in further improvement or better control of dewetting results; yet, the fundamental difference stated above suggests that the low-temp. annealing method cannot be combined with any of the other methods to facilitate NW catalysts by agglomeration. A combination of the three other methods might be useful in further reduction on NW catalyst diameters beyond lithography limits. 


\section{Summary}

We have demonstrated that combining selective area and lithography based catalystassisted approaches allows growing NWs of diameters considerably smaller than the initial catalyst diameter, which is dictated by the lithography process. The effect of the selective area mask on the metal catalyst was examined in two different growth methods; however, only by using SAVLS, disc-like catalysts of hundreds of nanometers in diameter, exhibited successful NW growth with 2-3 fold decrease of the resulting diameter. The generality of this concept was demonstrated by growing both InAs and InP NWs of different metallization thicknesses using this method, as well as NOSA based NWs of $\sim 20 \mathrm{~nm}$.

The diminishing diameter of the catalyst was modelled by assuming that the final catalyst shape is hemispherical; this assumption was found sufficient in describing the experimental results. We explain the effect of eliminating catalyst splitting by the selective area mask, in the confinement of the dewetted metallic disc to an area who's size is much smaller than the un-masked spreading area of a similar disc, thus increasing the chance of agglomeration. This result offers promise for bottom-up growth of sub-100 nm NWs by photolithography.

\section{Acknowledgements}

The financial support of the Russell Berrie Nanotechnology Institute (RBNI) and of the Israeli Nanotechnology Focal Technology Area on "Nanophotonics for Detection" (Grant 4369512) is highly appreciated. The fabrication was performed at the MicroNano Fabrication Unit (MNFU), Technion.

[1] Katsuhiro Tomioka, Masatoshi Yoshimura, and Takashi Fukui. A III-V nanowire channel on silicon for high-performance vertical transistors. Nature, 488(7410):189-192, 2012.

[2] Lin Chen, Wayne Y. Fung, and Wei Lu. Vertical Nanowire Heterojunction Devices Based on a Clean Si/Ge Interface. Nano Lett., 13(11):5521-5527, 2013.

[3] Peter Krogstrup, Henrik Ingerslev Jørgensen, Martin Heiss, Olivier Demichel, Jeppe V Holm, Martin Aagesen, Jesper Nygard, and Anna Fontcuberta i Morral. Single-nanowire solar cells beyond the Shockley-Queisser limit. Nature Photonics, 2013.

[4] Alberto Casadei, Esther Alarcon Llado, Francesca Amaduzzi, Eleonora Russo-Averchi, Daniel Rüffer, Martin Heiss, Luca Dal Negro, and Anna Fontcuberta i Morral. Polarization response of nanowires a la carte. Scientific reports, 5, 2015.

[5] Ziyuan Li, Xiaoming Yuan, Lan Fu, Kun Peng, Fan Wang, Xiao Fu, Philippe Caroff, Thomas P White, Hark Hoe Tan, and Chennupati Jagadish. Room temperature GaAsSb single nanowire infrared photodetectors. Nanotechnology, 26(44):445202, 2015.

[6] Lin Xu, Zhe Jiang, Quan Qing, Liqiang Mai, Qingjie Zhang, and Charles M Lieber. Design and Synthesis of Diverse Functional Kinked Nanowire Structures for Nanoelectronic Bioprobes. Nano Lett., 13(2):746-751, 2013.

[7] Ilaria Zardo, Sara Yazji, Nicolas Hörmann, Simon Hertenberger, Stefan Funk, Sara Mangialardo, Stefanie Morkötter, Gregor Koblmüller, Paolo Postorino, and Gerhard Abstreiter. E1 (A) 
Electronic Band Gap in Wurtzite InAs Nanowires Studied by Resonant Raman Scattering. Nano letters, 13(7):3011-3016, 2013.

[8] Daniel Rudolph, Lucas Schweickert, Stefanie Morkötter, Lukas Hanschke, Simon Hertenberger, Max Bichler, Gregor Koblmüller, Gerhard Abstreiter, and Jonathan J Finley. Probing the trapping and thermal activation dynamics of excitons at single twin defects in GaAs-AlGaAs core-shell nanowires. New Journal of Physics, 15(11):113032, 2013.

[9] Kimberly A. Dick. A review of nanowire growth promoted by alloys and non-alloying elements with emphasis on Au-assisted III-V nanowires. Prog. Cryst. Growth Charact. Mater., 54(3-4):138 173, 2008.

[10] Bernhard Mandl, Julian Stangl, Emelie Hilner, Alexei A. Zakharov, Karla Hillerich, Anil W. Dey, Lars Samuelson, Günther Bauer, Knut Deppert, and Anders Mikkelsen. Growth Mechanism of Self-Catalyzed Group III-V Nanowires. Nano Lett., 10(11):4443-4449, 2010.

[11] Kimberly A Dick and Philippe Caroff. Metal-seeded growth of III-V semiconductor nanowires: towards gold-free synthesis. Nanoscale, 6(6):3006-3021, 2014.

[12] Neil P Dasgupta, Jianwei Sun, Chong Liu, Sarah Brittman, Sean C Andrews, Jongwoo Lim, Hanwei Gao, Ruoxue Yan, and Peidong Yang. 25th Anniversary article: semiconductor nanowiressynthesis, characterization, and applications. Advanced Materials, 26(14):2137-2184, 2014.

[13] C. B. Collins, R. O. Carlson, and C. J. Gallagher. Properties of Gold-Doped Silicon. Phys. Rev., 105:1168-1173, Feb 1957.

[14] Thomas Mårtensson, Patrick Carlberg, Magnus Borgström, Lars Montelius, Werner Seifert, and Lars Samuelson. Nanowire Arrays Defined by Nanoimprint Lithography. Nano Lett., 4(4):699702, 2004.

[15] Aurélie Pierret, Moïra Hocevar, Silke L Diedenhofen, Rienk E Algra, E Vlieg, Eugene C Timmering, Marc A Verschuuren, George W G Immink, Marcel A Verheijen, and Erik P A M Bakkers. Generic nano-imprint process for fabrication of nanowire arrays. Nanotechnology, 21(6):065305, 2010.

[16] F Haas, K Sladek, A Winden, M von der Ahe, T E Weirich, T Rieger, H Lüth, D Grützmacher, Th Schäpers, and H Hardtdegen. Nanoimprint and selective-area MOVPE for growth of GaAs/InAs core/shell nanowires. Nanotechnology, 24(8):085603, 2013.

[17] Abdul Mazid Munshi, Dasa L Dheeraj, Vidar Tonaas Fauske, Dong-Chul Kim, Junghwan Huh, Johannes F Reinertsen, Lyubomir Ahtapodov, KD Lee, Babak Heidari, ATJ van Helvoort, et al. Position-Controlled Uniform GaAs Nanowires on Silicon using Nanoimprint Lithography. Nano Lett., 14(2):960-966, 2014.

[18] Law, Matt and Goldberger, Joshua and Yang, Peidong. Semiconductor nanowires and nanotubes. Annu. Rev. Mater. Res., 34:83-122, 2004.

[19] Hong Jin Fan, Peter Werner, and Margit Zacharias. Semiconductor nanowires: from selforganization to patterned growth. Small, 2(6):700-717, 2006.

[20] Maria E Messing, Karla Hillerich, Jessica Bolinsson, Kristian Storm, Jonas Johansson, Kimberly A Dick, and Knut Deppert. A comparative study of the effect of gold seed particle preparation method on nanowire growth. Nano Research, 3(7):506-519, 2010.

[21] Yonatan Calahorra, Yaakov Greenberg, Shimon Cohen, and Dan Ritter. Native-oxide-based selective area growth of InP nanowires via metal-organic molecular beam epitaxy mediated by surface diffusion. Nanotechnology, 23(24):245603, 2012.

[22] Dan Dalacu, Alicia Kam, D Guy Austing, Xiaohua Wu, Jean Lapointe, Geof C Aers, and Philip J Poole. Selective-area vapour-liquid-solid growth of InP nanowires. Nanotechnology, 20(39):395602, 2009.

[23] A Kelrich, Y Calahorra, Y Greenberg, A Gavrilov, S Cohen, and D Ritter. Shadowing and mask opening effects during selective-area vapor-liquid-solid growth of InP nanowires by metalorganic molecular beam epitaxy. Nanotechnology, 24(47):475302, 2013.

[24] Gabriele Bulgarini, Michael E Reimer, Maaike Bouwes Bavinck, Klaus D Jo?ns, Dan Dalacu, Philip J Poole, Erik PAM Bakkers, and Val Zwiller. Nanowire waveguides launching single 
photons in a Gaussian mode for ideal fiber coupling. Nano Lett., 14(7):4102-4106, 2014.

[25] Marijn AM Versteegh, Michael E Reimer, Klaus D Jöns, Dan Dalacu, Philip J Poole, Angelo Gulinatti, Andrea Giudice, and Val Zwiller. Observation of strongly entangled photon pairs from a nanowire quantum dot. Nature communications, 5, 2014.

[26] In all SEM images presented, any embedded text was digitally removed, while the scale bar was digitally enhanced; Figure 1 was false colored.

[27] Y Calahorra, Y Greenberg, S Cohen, and D Ritter. Catalyst design for native oxide based selective area InP nanowire growth. In Indium Phosphide and Related Materials (IPRM), 2012 International Conference on, pages 265-268. IEEE, 2012.

[28] T Mårtensson, M Borgström, W Seifert, B J Ohlsson, and L Samuelson. Fabrication of individually seeded nanowire arrays by vapour-liquid-solid growth. Nanotechnology, 14(12):1255, 2003.

[29] Linus E. Fröberg, Brent A. Wacaser, Jakob B. Wagner, Sören Jeppesen, B. Jonas Ohlsson, Knut Deppert, and Lars Samuelson. Transients in the Formation of Nanowire Heterostructures. Nano Lett., 8(11):3815-3818, 2008. PMID: 18811210.

[30] S Paiman, Q Gao, HH Tan, C Jagadish, K Pemasiri, M Montazeri, HE Jackson, LM Smith, JM Yarrison-Rice, X Zhang, et al. The effect of V/III ratio and catalyst particle size on the crystal structure and optical properties of InP nanowires. Nanotechnology, 20(22):225606, 2009.

[31] We do not further emphysize the role of the native oxide in improving agglomeration, and assume that any type of close-fitted mask would result in a similar effect. In case of deposited mask materials, a careful dry etching stage, followed by a short wet etch, would be helpful in achieving better confinement, compared to full wet etch which results in an annular opening of the mask around the catalyst.

[32] As stated, this report mainly deals with NW diameter control. The length-diameter relations in this growth regime have been discussed previously [23]. Indeed due to the large diameter, the NWs reported here are relatively short.

[33] David J Srolovitz and Max G Goldiner. The thermodynamics and kinetics of film agglomeration. JOM, 47(3):31-36, 1995.

[34] E. Dornel, J-C. Barbé, F. de Crécy, G. Lacolle, and J. Eymery. Surface diffusion dewetting of thin solid films: Numerical method and application to $\mathrm{Si} / \mathrm{SiO}_{2}$. Phys. Rev. B, 73:115427, Mar 2006.

[35] Denis Gentili, Giulia Foschi, Francesco Valle, Massimiliano Cavallini, and Fabio Biscarini. Applications of dewetting in micro and nanotechnology. Chem. Soc. Rev., 41:4430-4443, 2012.

[36] Carl V Thompson. Solid-state dewetting of thin films. Annu. Rev. Mater. Res., 42:399-434, 2012.

[37] SJ Henley, JD Carey, and SRP Silva. Pulsed-laser-induced nanoscale island formation in thin metal-on-oxide films. Physical Review B, 72(19):195408, 2005.

[38] Jongpil Ye and Carl V Thompson. Templated solid-state dewetting to controllably produce complex patterns. Advanced Materials, 23(13):1567-1571, 2011.

[39] F Ruffino, A Pugliara, E Carria, L Romano, C Bongiorno, C Spinella, and MG Grimaldi. Novel approach to the fabrication of au/silica core-shell nanostructures based on nanosecond laser irradiation of thin au films on si. Nanotechnology, 23(4):045601, 2012.

[40] F Ruffino and MG Grimaldi. Template-confined dewetting of au and ag nanoscale films on mica substrate. Applied Surface Science, 270:697-706, 2013.

[41] T Baron, M Gordon, F Dhalluin, C Ternon, P Ferret, and P Gentile. Si nanowire growth and characterization using a microelectronics-compatible catalyst: PtSi. Appl. Phys. Lett., 89(23):233111-233111, 2006.

[42] Yue Wu, Yi Cui, Lynn Huynh, Carl J Barrelet, David C Bell, and Charles M Lieber. Controlled growth and structures of molecular-scale silicon nanowires. Nano Lett., 4(3):433-436, 2004.

[43] Claudia Manuela Müller and Ralph Spolenak. Determination of the au/sin x interfacial energy by afm and fib tomography. Surface Science, 617:94-105, 2013.

[44] I Beszeda, IA Szabo, and EG Gontier-Moya. Morphological evolution of thin gold films studied by auger electron spectroscopy in beading conditions. Applied Physics A, 78(7):1079-1084, 2004.

[45] D Amram, L Klinger, and E Rabkin. Anisotropic hole growth during solid-state dewetting of 
single-crystal au-fe thin films. Acta Materialia, 60(6):3047-3056, 2012.

[46] Anna Kosinova, Oleg Kovalenko, Leonid Klinger, and Eugen Rabkin. Mechanisms of solid-state dewetting of thin au films in different annealing atmospheres. Acta Materialia, 83:91-101, 2015.

[47] Dor Amram, Oleg Kovalenko, Leonid Klinger, and Eugen Rabkin. Capillary-driven growth of metallic nanowires. Scripta Materialia, 109:44-47, 2015.

[48] A Sundar, RA Hughes, P Farzinpour, KD Gilroy, GA Devenyi, JS Preston, and S Neretina. Manipulating the size distribution of supported gold nanostructures. Appl. Phys. Lett., 100(1):013111, 2012.

[49] Pouyan Farzinpour, Aarthi Sundar, Kyle D Gilroy, Zachary E Eskin, Robert A Hughes, and Svetlana Neretina. Dynamic templating: a large area processing route for the assembly of periodic arrays of sub-micrometer and nanoscale structures. Nanoscale, 5(5):1929-1938, 2013.

[50] Dong Pan, Mengqi Fu, Xuezhe Yu, Xiaolei Wang, Lijun Zhu, Shuaihua Nie, Siliang Wang, Qing Chen, Peng Xiong, Stephan von Molnár, et al. Controlled synthesis of phase-pure InAs Nanowires on Si (111) by diminishing the diameter to $10 \mathrm{~nm}$. Nano Lett., 14(3):1214-1220, 2014.

[51] Pramod Nimmatoori, Qi Zhang, Elizabeth C Dickey, and Joan M Redwing. Suppression of the vapor-liquid-solid growth of silicon nanowires by antimony addition. Nanotechnology, 20(2):025607, 2009. 